\title{
Patient-Reported Outcome Is Important in Psychosocial Intervention for Dementia: A Secondary Analysis of a Randomized Controlled Trial of Group Reminiscence Approach Data
}

\author{
Kenichi Meguro Kyoko Akanuma Mitsue Meguro \\ Department of Geriatric Behavioral Neurology, Tohoku University Graduate School of Medicine, \\ Sendai, Japan
}

We have previously evaluated the beneficial effect of the group reminiscence approach (GRA) in patients with vascular dementia (VaD) in respect of cognitive and observed behavioral parameters [1]. Sixty patients who had been diagnosed with VaD were randomly assigned to three arms: a GRA arm, a social contact (SC) arm, and a control arm. A 1-hour session of GRA and SC was held once a week for 3 months with the GRA and SC arms, respectively. Only supportive care was given to those in the control arm. An improvement in cognitive function and behavioral activities was defined as the primary outcome. However, we found that no significant improvements in the main outcome measures had been demonstrated and concluded that these results did not support the hypothesis that GRA is beneficial to patients with VaD.

As reported, the dropout ratio was higher in the SC arm $(9 / 20=45 \%)$ compared with the GRA arm $(3 / 20=15 \%)$ or the control arm $(3 / 20=15 \%)$. The participants in the SC arm told us that they had found that the intervention was very boring. In the case of the SC arm, the group was asked during a 50-min session to talk about social issues such as 'health and disease management' or 'hot topics from today's newspaper', etc., as well as interests in daily life such as 'recreational activities in their facilities' or 'meals served in their facilities', etc. The discourse of older adults easily moved to their memories of the past as was the case in the GRA arm, whereas when their talk was blocked off by presenting social topics, participants in the SC arm appeared to be bored.

Having assessed emotional ratings in the GRA and SC arms, we could proceed to a secondary analysis. We had assessed 5 ratings: 'very enjoyable', 'enjoyable', 'normal', 'boring', and 'very boring'. In the GRA arm, 13/17 (=76\%) answered 'very enjoyable' or 'enjoyable', whereas in the SC arm, 3/11 (=27\%) answered in this way. We thus operationally classified them into two groups: the 'enjoyable' group $(n=16$, i.e., 13 in the GRA arm plus 3 in the SC 
arm) and the 'boring' group ( $\mathrm{n}=12$, i.e., 4 in the GRA arm plus 8 in the SC arm), independent of 'outer' factors such as the GRA or SC groups. As described previously [2], the standard deviation of changes over 21 days was 5.01 in the placebo arm according to the Multidimensional Observation Scale for Elderly Subjects (MOSES). A decrement greater than the standard deviation multiplied by 1.96, i.e., 10 or more on the MOSES, was defined as improvement.

We found that the 'enjoyable' group compared with the 'boring' group showed a statistically significant improvement on the MOSES $(\mathrm{p}<0.05)$.

We have previously reported a dementia case that showed an improved inner mental world after using a psychosocial approach, even though the change in the MMSE was unremarkable [3]. Unlike the clinical trials of drugs, psychosocial approaches may not be adequate for the randomized controlled trial design, and we should be cautious about 'superficial' interpretations. Nevertheless, patient-reported outcomes [4] may be important for psychosocial intervention.

\section{References}

1 Ito T, Meguro K, Akanuma K, Ishii H, Mori E: A randomized controlled trial of the group reminiscence approach in patients with vascular dementia. Dement Geriatr Cogn Disord 2007;24:48-54.

2 Allain H, Dautzenberg P, Maurer K, Schuck S, Bonhomme D, Gerard D: Double blind study of tiapride versus haloperidol and placebo in agitation and aggressiveness in elderly patients with cognitive impairment. Psychopharmacology 2000;148:361-366.

3 Meguro M, Meguro K: Activated thalamic glucose metabolism after combined donepezil and psychosocial intervention. Br J Neurosci Nurs 2010;6:176-180.

4 Frank LB: Commentary on 'Health economics and the value of therapy in Alzheimer's disease.' Report from the Alzheimer's Association Research Roundtable on patient-reported outcomes and dementia research. Alzheimers Dement 2007;3:162-165. 